\title{
LA ENSEÑANZA DE LAS CIENCIAS DESDE EL ENFOQUE CTSA EN EL SECTOR PROVINCIAL COLOMBIANO
}

\author{
O Ensino das Ciências da Abordagem CTSA no Setor Provincial Colombiano
}

\author{
Omar Alejandro Benites Rozo ${ }^{1}$
}

\begin{abstract}
Resumen: La enseñanza de las ciencias desde el enfoque Ciencia, Tecnología, Sociedad y Ambiente (CTSA) ha sido implementada en las instituciones educativas en Colombia desde que el Ministerio de Educación Nacional (MEN) publicó en el 2004 los estándares curriculares, sin embargo, tanto el seguimiento de la implementación del enfoque CTSA en los diseños curriculares, así como el proceso de enseñanza aprendizaje de las ciencias naturales de manera contextualizada no han recibido la atención necesaria. Esos dos elementos han ampliado significativamente la brecha, a nivel de calidad educativa que existe entre el sector urbano y el rural. De esa manera, se presenta el siguiente documento que pretende desarrollar un debate frente a la necesidad de desarrollar procesos de educación en ciencias pertinentes y contextualizados, para lo cual aborda dos aspectos de manera general. El primero corresponde a una nueva categoría territorial del país que requiere ser atendida desde las políticas educativas y las comunidades de especialistas, la cual corresponde a un espacio territorial denominado sector provincial, el cual cuenta con una serie de características propias de la ruralidad nacional. Por otro lado, se abordan los aspectos más representativos del enfoque CTSA, especialmente los que corresponden con las propuestas estatales, con la intención de dar fundamentación teórica al último apartado del documento, en el cual se propone una articulación entre el enfoque CTSA y la educación en ciencias en el sector provincial.
\end{abstract}

Palabras clave: Sector Provincial. Enseñanza de las Ciencias. Enfoque CTSA. Educación Contextualizada.

Resumo: O ensino de ciências da abordagem da Ciência, Tecnologia, Sociedade e Meio Ambiente (CTSA) foi implementado em instituições de ensino na Colômbia desde que o Ministério da Educação Nacional (MEN) publicou em 2004 os padrões curriculares, no entanto o seguimento da implementação da abordagem CTSA nos desenhos curriculares, bem como o processo de ensino da aprendizagem de ciências naturais de maneira contextualizada, não receberam a atenção necessária. Esses dois elementos aumentaram significativamente a diferença, no nível de qualidade educacional que existe entre os setores urbano e rural. Dessa forma, é apresentado o seguinte documento, que pretende desenvolver um debate sobre a necessidade de desenvolver processos relevantes e contextualizados de educação científica, para os quais aborda dois aspectos de maneira geral. O primeiro corresponde a uma nova categoria territorial do país que precisa ser

\footnotetext{
${ }^{1}$ Doctorando en Educación de la Universidad Pedagógica Nacional. Docente de Ciencias de la I.E.D Sabio Mutis en el municipio de La Mesa Cundinamarca Colombia. Orcid: 0000-0001-9259-6654. Correo electrónico: oabenitezr@hotmail.com - oabeitezr@upn.edu.co. Con este documento se pretende dar cumplimiento con un examen de candidatura desde el énfasis de ciencias, para optar al título de Doctor en Educación Doctorado en Educación - Universidad Pedagógica Nacional (DIE - UPN).
} 
atendida por políticas educacionais e comunidades de especialistas, que corresponde a um espaço territorial denominado setor provincial, que possui uma série de características típicas da ruralidade nacional. Por outro lado, são abordados os aspectos mais representativos da abordagem da CTSA, especialmente aqueles que correspondem às propostas estaduais, com o intuito de fundamentação teórica da última seção do documento, na qual é proposta uma articulação entre a abordagem da CTSA e a educação em ciências no setor provincial.

Palavras-chave: Setor Provincial. Ensino de Ciências. Abordagem CTSA. Educação Contextualizada.

\section{Introducción}

La educación en el contexto Colombiano se desarrolla fundamentalmente en dos escenarios: el primero atiende las necesidades de los jóvenes que habitan las grandes ciudades y el segundo corresponde a atender las necesidades de los jóvenes que viven en el resto del país. Esa clasificación ha existido durante varias décadas y ha generado situaciones particulares de los contextos sociales como una dicotomía entre la educación de los contextos urbanos y rurales, brechas particularmente marcadas en cuanto a la calidad, impacto, pertenencia y capacitación docente, entre otros aspectos.

Aunque existen investigaciones aisladas que han intentado atender las características de los territorios rurales del país, vale la pena precisar que en la actualidad no existe una comunidad investigativa que atienda las necesidades propias de ese sector. Ese desconocimiento no es diferente frente al diseño, publicación y ejecución de políticas públicas a nivel educativo en cada una de las áreas del conocimiento.

Sin embargo, durante el proceso de consolidación de los acuerdos de paz entre el Estado y las Fuerzas Armadas Revolucionarias de Colombia (FARC), se propuso diseñar y ejecutar un programa especial para la educación rural, el cual no solo atendiera las necesidades educativas de dicho contexto, sino que también permitiera disminuir la brecha que existe entre los dos grandes espacios territoriales del país. Es así como durante el 2017, en cabeza del Ministerio de Educación Nacional se diseñó el Plan Especial de Educación Rural (PEER), convirtiéndose ese documento en la primera aproximación del Estado colombiano de atender las necesidades de la educación rural dispersa del país.

Es necesario aclarar que el programa solo atiende el contexto rural disperso que ha sido afectado por el conflicto armado, dejando de lado aquellos escenarios que se enmarcan en un espacio intermedio entre lo urbano y lo rural, el cual vale la pena resaltar que aproximadamente el 68,7\% de los municipios del país se encuentran en esta categoría, que de ahora en adelante se denominará Contexto Provincial, en el que convergen dos realidades socioculturales: la de las familias que viven en las cabeceras municipales y la de aquellas que habitan los espacios territoriales rurales.

Es ese escenario que pretende ser abordado en este documento, especialmente frente a los aspectos más generales que requiere la enseñanza de las ciencias desde el enfoque Ciencia, Tecnología, Sociedad y Ambiente (CTSA). Es de resaltar que existen aisladas investigaciones que atienden las particularidades del sector provincial y que involucran la enseñanza de las ciencias desde el enfoque CTSA. 
Las reflexiones anteriores son un punto de partida para invitar a las comunidades de especialistas a iniciar procesos investigativos en la enseñanza de las ciencias en el sector provincial colombiano.

\section{La provincia: un escenario por explorar}

Uno de los autores que mayores aportes realizó al estudio de la provincia fue Orlando Fals Borda, que en varios textos defendió la viabilidad de la provincia en el ordenamiento territorial colombiano. De igual forma estudia la entidad territorial de las provincias y establece la importancia de esta forma de administración local como respuesta al modelo globalizador y de mercado y a "las transformaciones tecnológicas y multiviolencias" (FALS-BORDA, 2000) que padece Colombia. En ese sentido, expone el papel que juega la provincia, llamada también subregión, como coordinadora de municipios que tienen intereses en común y la que permitiría dar solución a los diferentes conflictos sociales, educativos y económicos que padece el país, pues permitiría mayor gobernabilidad.

A pesar de los acercamientos conceptuales que se han hecho a la provincia como entidad territorial, también ha sido entendida de manera peyorativa, puesto que en ocasiones refleja atraso y retroceso sociocultural. Por tanto, la problemática reside en los usos y los desusos de lo que significa la provincia. Con el devenir histórico de nuestro país, ha pasado a ser percibida, en algunos departamentos, como una entidad alegórica que adquiere valor por su legado histórico y cultural, pero no por la utilidad que estas pueden tener para el desarrollo social y económico del país si se le asignan funciones claras y que en últimas hace referencia a aquello que quedó como herencia de un pasado exclusivamente rural y ajeno a los avances del desarrollo urbano (PÉREZ et al., 1998).

En Colombia las provincias son relevantes para el estudio de los patrones de ruralidad, ya que impulsan una concepción territorial por encima de la administración y provisión de los servicios públicos y más hacia la provisión de herramientas y modos de generación de ingreso, conocimiento, innovación y aprendizaje propio o en subredes de cooperación y competencia. Además, la escala geográfica es una consideración clave en la definición de urbanización y ruralidad: si el marco de referencia comprende territorios pequeños como ciudades o áreas metropolitanas, será más evidente el concepto de urbanización, mientras que la ruralidad será un concepto relevante si el marco de referencia comprende territorios grandes (TORRE; WALLET, 2016). Lo mencionado anteriormente es una realidad cercana al país. Según la división territorial oficial del Departamento Nacional de Estadística (DANE), Colombia se divide en 141 provincias, sin embargo, esa clasificación no se usa de manera político-administrativa, sino que sirve para propósitos de recolección de información y, en ciertos departamentos, se mantiene su vigencia para la zonificación y administración de algunos servicios públicos.

Así mismo, es evidente que el concepto de provincia toma bastante fuerza en la medida en que se convierte en un espacio territorial donde convergen intereses económicos, políticos, sociales, culturales y educativos de diferentes municipios. Por lo tanto, se hace necesario que el sistema educativo se empiece a pensar desde los elementos representativos de la provincia y de esta manera se descentralicen un poco las políticas educativas, con el fin de darle reconocimiento a las características propias de las provincias. Los aspectos mencionados anteriormente abren una pregunta importante a la hora de desarrollar procesos pedagógicos en el sector provincial, en especial desde la enseñanza de las ciencias. Esta pregunta se desarrolla en el siguiente apartado. 


\section{1 ¿Qué requiere la educación provincial?}

Los procesos educativos e investigativos realizados en las instituciones que se encuentran ubicadas en los municipios que hacen parte de las provincias no solo requieren repensar las políticas públicas o la investigación por parte de la comunidad de especialistas, sino que también se hace necesario caracterizar el rol del maestro y del estudiante. En esta dirección apunta esta investigación: el maestro de provincia demanda que la formación en investigación sea una actividad inherente a toda actividad humana intencional (POPHAM, 1990). Por lo tanto, la escuela provincial requiere orientarse a prácticas pedagógicas que le permitan al estudiante ser competente dentro de la universalización o globalización de la cultura, pero que a su vez le aporte elementos de identidad y reconocimiento de su entorno como pilar de su formación y como elemento que contribuye a la construcción de una sociedad cada vez más educada. Eso se puede lograr si los procesos de enseñanza se hacen desde el diseño de estrategias pedagógicas que involucren directamente el contexto de la comunidad educativa (HERNÁNDEZ, 2001).

Por otro lado el estudiante de la provincia cuenta con una interacción directa con la naturaleza, dichos elementos le permiten enriquecer su concepción sobre la ciencia y sus respectivas aplicaciones, de igual forma cuenta con el conocimiento que adquiere fuera de la escuela de manera experiencial, vivencial, anecdótica informal y relevante.

Para que la enseñanza de las ciencias sea coherente con el sector provincial, se requiere, se propone que los procesos pedagógicos se construyan sobre las experiencias informales de los estudiantes, que suceden previa o paralelamente a los aprendizajes escolares (ZAMBRANO, 1998). Es decir, las relaciones entre el conocimiento común y el conocimiento científico que, en el proceso de aprendizaje escolar corresponderían a las relaciones entre el conocimiento previo de los estudiantes y el conocimiento escolar impartido por el maestro, pueden darse, al menos, en tres formas fundamentales, como se menciona a continuación (HERNÁNDEZ, 2001).

a) Cuando los conocimientos se interrelacionan como similares, la enseñanza aparece como adaptación;

b) Cuando la forma de interrelación entre elementos distintos no se revela como un conflicto, la enseñanza aparece como integración;

c) Cuando se plantean oposiciones entre el conocimiento previo y el conocimiento escolar, la enseñanza aparece como un intercambio de posiciones.

Desde esa perspectiva, el diseño metodológico que se implemente en las instituciones educativas que hagan parte de la provincia deben favorecer la interacción de estos dos escenarios que convergen en la provincia (Intermedio y rural), permitiendo una producción colectiva de conocimientos, así como la indagación de las necesidades específicas del entorno para innovar y encontrar soluciones. A través de esta investigación se propone dar cuerpo a la discusión de la educación en el sector provincial, aportando elementos como la importancia de un diálogo permanente de saberes con la comunidad, reflexionando sobre su realidad y las implicaciones que requieren ser pensadas. El esquema 1 permite ver como el escenario educativo provincial se convierte en una oportunidad pedagógica para las escuelas ubicadas en los sectores intermedios y rurales, debido a que en esos espacios académicos convergen dos realidades socioculturales con diversas experiencias, expectativas, vivencias, entre otras. Por un lado está todo lo que 
aportan los estudiantes que viven en los municipios o cabeceras municipales; por otro, se encuentra lo aportado por aquellos estudiantes que viven en el campo.

Esquema 1 - Representación del escenario educativo de la provincia
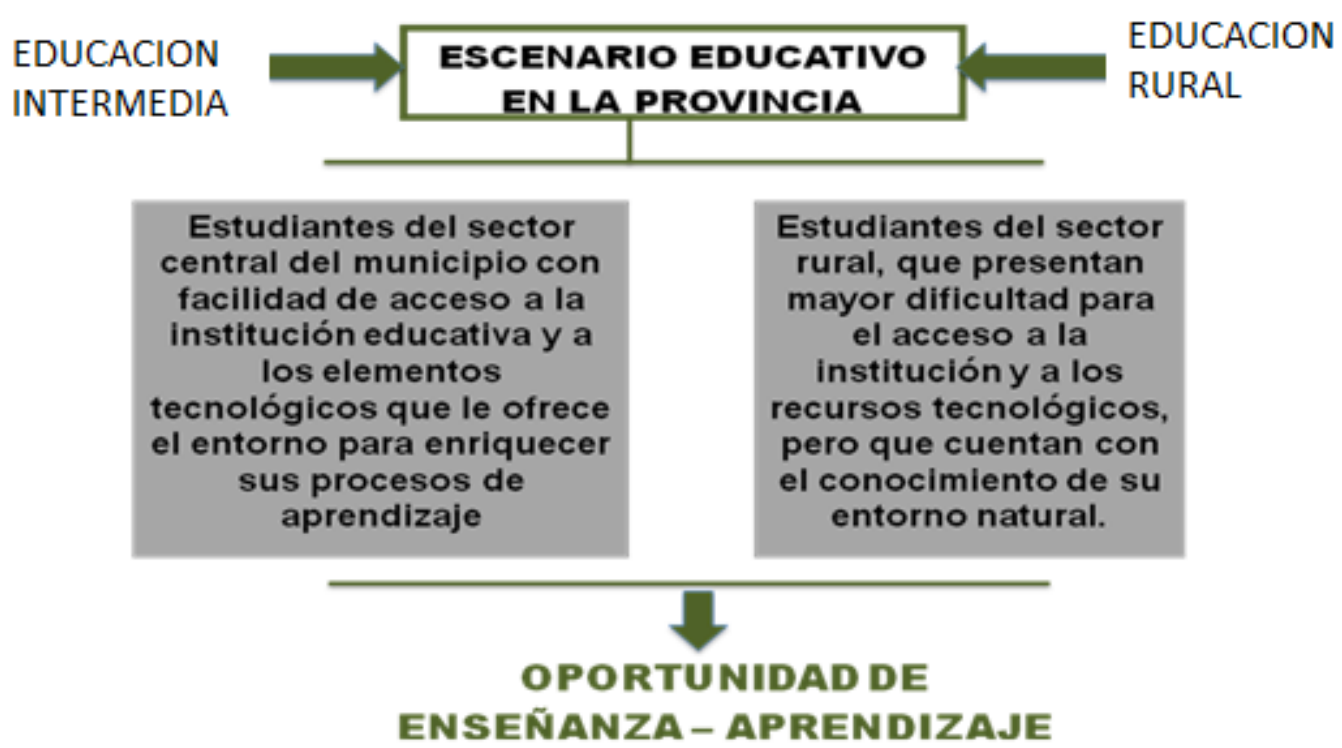

En esta imagen se muestra la posible relación que puede existir entre la educación intermedia y la rural. Ajustado de: Leer la provincia para construcción de saber. II congreso de Transformación Educativa (MARTÍN, 2015).

Dentro de las investigaciones encontradas frente al sector rural, está la propuesta para la educación en ciencias en el sector rural (MARTIN, 2009), en la cual se afirma que la escuela debe apuntar hacia la reconstrucción de las formas de significar y actuar; hacia el reconocimiento de que el estudiante ya posee unas formas de significar y actuar sobre el mundo o el ambiente natural o social que lo rodea. Por lo tanto, al pensar estrategias pedagógicas en el sector provincial, se requeriría que estas atendieran a: las realidades de la provincia, las formas de representar el mundo, la diversidad como el escenario del aprendizaje y la motivación de procesos de cooperación y construcción colectiva del conocimiento para así generar transformaciones que reconozcan lo local como punto de encuentro y desde las identidades locales insertarse en la universalización del conocimiento (MARTÍN, 2015). Dentro de las conceptualizaciones que se han realizado en torno a la influencia del contexto de los estudiantes en su proceso de aprendizaje. Así mismo, desde esa perspectiva podemos resaltar la propuesta de Freire en torno a la acciónparticipación, que permite entender que en la provincia la transición del conocimiento, evidenciada en el constante diálogo de saberes, permite apostar por unos ciudadanos críticos, capaces de novedad en el conocimiento y transformadores de su entorno. Esos aspectos abren el debate sobre la necesidad de desarrollar un proceso de enseñanza de las ciencias de manera contextualizada y en ese sentido atender las necesidades y características del sector provincial Colombiano, especialmente porque este abarca más del $50 \%$ del territorio nacional. 


\section{Enseñanza de las ciencias naturales en el sector provincial colombiano}

En este apartado se va hacer referencia a lo correspondiente frente a la enseñanza de las ciencias, específicamente los aspectos que tengan alguna relación con el sector provincial colombiano. En ese orden de ideas, se profundizarán aspectos como: fines de la educación en ciencias en Colombia, características del enfoque Ciencia Tecnología Sociedad y Ambiente (CTSA), para finalmente poder fundamentar teóricamente la importancia de este trabajo desde la enseñanza de las ciencias naturales en el sector provincial.

\subsection{Propósitos de la educación en ciencias naturales en Colombia}

Entre los fines de la educación en ciencias naturales en Colombia se resalta el carácter de construcción humana de la ciencia con la intención de mostrar que al reconocerla de esta forma, se tiene que aceptar la necesidad de concebir de una forma diferente la enseñanza de las ciencias, por lo tanto, no se trata de transmitir verdades inmutables, sino de darle al estudiante la posibilidad de ver que su perspectiva del mundo no es el mundo, sino una perspectiva de él (MINISTERIO DE EDUCACIÓN NACIONAL, 1994).

Enseñar ciencias en cualquier institución educativa debe consistir en darle al estudiante la oportunidad de establecer un diálogo racional entre su propia perspectiva del mundo, la de sus compañeros y el conocimiento abordado en el aula de clases, en palabras de Furió y Vilches, la alfabetización científica significará que la gran mayoría de la población dispondrá de los conocimientos científicos y tecnológicos necesarios para desenvolverse en la vida diaria, ayudar a resolver los problemas y necesidades de salud y supervivencia básicos, tomar conciencia de las complejas relaciones entre ciencia y sociedad y, en definitiva, considerar la ciencia como parte de la cultura de nuestro tiempo (FURIÓ; VILCHES, 1997).

En concordancia con lo anterior, se hace necesario destacar la estrecha relación que existe entre el conocimiento cotidiano y el conocimiento escolar. Este último ha sido motivo de estudio de diferentes autores, que lo definen de la siguiente manera: desde una perspectiva sociológica, el conocimiento escolar se refiere al conjunto de saberes y prácticas que distribuye comunicativamente la escuela a distintos grupos sociales a través de su propio funcionamiento o dispositivo pedagógico (BERNSTEIN, 1996). Por otro lado, si se quiere abordar el conocimiento escolar desde una perspectiva sociológica, curricular y didáctica, se pueden reconocer definiciones de este que lo caracterizan como un saber social, que es organizado en el currículo escolar y transformado para ser enseñado en el aula (GRANATA; BARALE; CHADA, 2005), promoviendo determinados procesos cognitivos de carácter individual y colectivo en los estudiantes, cuya cristalización pedagógica el saber social e institucionaliza el conocimiento (CASTAÑEDA; ROSAS; MOLINA, 2012) y constituye el aprendizaje escolar.

Por otro lado, el conocimiento cotidiano es propio de las experiencias y reflexiones diarias de las personas. Este no requiere específicamente de experimentación para que se dé, sino que se puede dar a partir de reflexiones y discusiones que se dan día a día entre los integrantes de una familia o una comunidad, algunas características de conocimiento cotidiano son: que es y ha sido respuesta de necesidades vitales, también que ofrece resultados prácticos y que se puede transmitir de generación en generación. En esta 
investigación, la provincia colombiana es el escenario que brinda el conocimiento cotidiano.

De acuerdo a lo anterior, hay propiedades que distinguen al conocimiento científico del cotidiano. La primera y más importante es la intención teórica del científico. Todo científico intenta hacer teorías de gran generalidad acerca de lo que quiere conocer. Cuanto más general sea una teoría, más importancia tendrá dentro del contexto de la comunidad de científicos dedicados a esa área de conocimiento. El conocimiento común, por el contrario, no se preocupa por la construcción de teorías que vinculen hechos y procesos aparentemente sin ninguna relación. El conocimiento del hecho o del proceso mismo es ya satisfactorio (MEN, 1994). En otras palabras, el conocimiento de algo nuevo no satisface enteramente la curiosidad del científico o del tecnólogo sino que, por el contrario, les plantea muchísimos nuevos problemas que solo se resolverán con la construcción de una gran teoría capaz de explicar la relación entre los fenómenos en cuestión y muchas otras relaciones conocidas y otras que se pondrán en evidencia gracias a la construcción de la teoría misma.

En relación con lo anterior es importante aclarar que uno de los grandes problemas de los programas curriculares colombianos es la falta de perspectiva histórica que permita relativizar sanamente la concepción de realidad y la de verdad. Cuando se piensa en la verdad como absoluta o en la realidad como algo independiente de la comunidad científica que la concibe, se está asumiendo que la ciencia ya no puede evolucionar. Pero, de acuerdo a lo aceptado por las comunidades de especialistas, la realidad es una representación de lo real mediante un modelo (o una metáfora). El estudiante normalmente cree que la realidad es como se dice en los libros que es. Pocas veces el estudiante es consciente de que lo que estudia en los libros son diversos modelos que algún día pueden ser superados por otros, mucho menos es consciente de que esos modelos son construcciones sociales (culturales) en las que él algún día puede participar.

Una sugerencia metodológica importante es permitir que los estudiantes actúen según este impulso natural y darles el tiempo necesario para que ofrezcan sus propios modelos o sus metáforas para dar cuenta de una cierta realidad. Los estudiantes, si se les da la oportunidad, ofrecen fácilmente modelos bastante interesantes y que en su entorno escolar se pueden consolidar en una riqueza intelectual.

Por lo tanto, el secreto estaría en proporcionar las condiciones para que los modelos ofrecidos por los estudiantes evolucionen. La evolución de esos modelos, que ellos percibirán de una forma muy comprometida, no solo les permitirá entender la versión "actualizada" del modelo, sino que además no perderán de vista ese carácter evolutivo de la construcción científica. El educando entiende la estructura del conocimiento científico y la forma como se construye y no se limita a memorizar algunos de los resultados logrados en un determinado momento de la historia de la ciencia (MEN, 1998).

En consecuencia, una de las misiones de la escuela es construir, vivificar y consolidar valores fortaleciéndolos desde el uso del contexto sociocultural de los estudiantes. De esta manera, dicho espacio académico es responsable de aprovechar el conocimiento común y las experiencias previas de los estudiantes como uno de los insumos en la enseñanza de las ciencias, de tal manera que el aprendizaje que se desarrolla en las aulas pueda ser puesto al servicio de la comunidad (MEN, 1994).

Es así que la política educativa, el currículo en general y la escuela como institución, no deben ser ajenas a la problemática social que generan la ciencia y la 
tecnología y su influencia en la cultura y en la sociedad. Por tal razón, la escuela debe tomar como insumo las relaciones que se dan entre ciencia, tecnología, sociedad, cultura y medio ambiente, con el fin de reflexionar no solo sobre sus avances y uso, sino también sobre la formación y desarrollo de mentes creativas y sensibles a los problemas, lo cual incide en la calidad de vida del hombre y en el equilibrio natural del medio ambiente (MEN, 2006).

La escuela en cuanto sistema social y democrático, debe educar para que los individuos y las colectividades comprendan la naturaleza compleja del ambiente, resultante de la interacción de sus aspectos biológicos, físicos, químicos, sociales, económicos y culturales; construyan valores y actitudes positivas para el mejoramiento de las interacciones hombre-sociedad naturaleza, para un manejo adecuado de los recursos naturales y para que desarrollen las competencias básicas y así resolver problemas ambientales (MEN, 1994). Por lo tanto, si la escuela es autónoma para elaborar y llevar a cabo participativamente su propio PEI, entonces el currículo debe responder a los problemas, intereses, necesidades y aspiraciones del alumno y de la comunidad y a la política educativa nacional.

La organización escolar implica que la comunidad educativa haya llegado a un consenso en cuanto a los grandes logros educativos que se desean alcanzar, además de establecer un "clima propicio para el aprendizaje". Eso quiere decir que la escuela debe organizar y facilitar el aprendizaje del estudiante ayudándole a abordar problemas de interés, realizando seguimiento a las actividades emprendidas por él, facilitando así su autonomía y creatividad y orientándolo en la búsqueda de información (bibliografía, centros de documentación, especialistas y prácticos, investigadores, laboratorios, etc.). Esos aspectos de la enseñanza son abordados en Colombia desde los planteamientos realizados desde el Ministerio de Educación Nacional (MEN) en las diferentes políticas educativas, sin embargo, dichos procesos tienen una sola herramienta de seguimiento que son las pruebas Saber, que se aplican a los estudiantes de los grados 11, es decir que, desde los procesos planteados por el Estado colombiano, existe una mínima atención a las necesidades, características e impacto de la inclusión del enfoque CTSA en los estándares curriculares y su respectiva planeación a nivel institucional.

\subsection{Fundamentos del enfoque CTSA}

El enfoque CTSA es un campo de investigación que permite al estudiante comprender el impacto de la ciencia y la tecnología en el contexto social y se consolida como una propuesta educativa innovadora de carácter general con la finalidad de promover la formación en conocimientos científicos y especialmente en valores que favorezcan la participación ciudadana en la evaluación y el control de las implicaciones sociales y ambientales (MARTÍNEZ; ROJAS, 2006). Dentro de las propuestas del profesor Martínez, se evidencia que así como en otros modelos educativos, cada actor tiene un rol especial en el proceso de enseñanza aprendizaje de las ciencias.

\subsection{Enfoque CTSA: un aporte al currículo desde la enseñanza de las ciencias}

La enseñanza de las ciencias ha sido tema de discusión y debate en las diferentes comunidades de investigadores del sector educativo. Dentro de dichos debates, durante las últimas décadas ha tomado fuerza lo relacionado con el impacto que pueden tener las relaciones CTSA, en la enseñanza de las ciencias, atendiendo profundamente las 
necesidades de la sociedad actual. En especial, dicha preocupación se ha centrado en buscar herramientas que permitan fortalecer los currículos para la formación a diferentes niveles, o cambiarlos mediante reformas educativas (ACEVEDO, 1995, 1996; GIL et al., 2003; MAIZTEGUI et al., 2002; MANSOUR, 2010; SANMARTIN et al., 1992; SOLBES, 2002). Algunas recomendaciones derivadas de sus investigaciones se encaminan a (TORO, 2014):

a) La capacidad de realizar evaluaciones sobre diversos avances científicos y tecnológicos, su utilidad, sus riesgos y su impacto social y ambiental;

b) Valorar y generar juicios éticos sobre los desarrollos tecnológicos encaminados a la satisfacción de necesidades humanas y a la solución de los problemas del mundo;

c) Propiciar una mayor participación social, a través del análisis, la discusión y puesta en marcha de las decisiones y actuaciones de la comunidad frente a los adelantos o problemáticas mundiales y regionales.

Según Solbes e Vilches (2004), cumplir con esos objetivos posibilitaría formar desde la escuela ciudadanos científicamente alfabetizados. Un ejemplo de esta intención fue lo que sucedió en Estados Unidos en la reforma educativa de 1991, la cual tenía como meta principal para el siglo XXI usar la enseñanza y el aprendizaje de la ciencia / tecnología en el contexto de la experiencia humana, con el fin de "formar estudiantes con destrezas que les permitiera convertirse en ciudadanos activos y responsables para afrontar las distintas problemáticas que afectaran sus vidas".

Esa preocupación impactó de manera directa el currículo Colombiano. Las reflexiones y discusiones han trascendido a escenarios académicos y, más importantes aún, políticos. Es así como en el año 1994 el Ministerio de Educación Nacional (MEN) publicó los Estándares Básicos en competencia de ciencias naturales y sociales, que partieron de los lineamientos generales de educación de 1998. Para el caso específico de ciencias naturales, en el documento del MEN se observan como aporta tres grandes acciones de pensamiento que son: (a) la manera de aproximarse al conocimiento como lo hacen los científicos y las científicas; (b) el manejo de los conocimientos propios de las ciencias naturales y (c) el desarrollo de compromisos personales y sociales.

Para el caso del fortalecimiento disciplinar, el MEN propone un esquema por cada nivel, en el que clasifica los conocimientos propios de las ciencias naturales en tres columnas, que son: entorno vivo, entorno físico y ciencia, tecnología y sociedad. Es importante resaltar que la inclusión del enfoque CTS con un componente ambiental, desde cada una de las áreas abordadas en el diseño curricular de las ciencias naturales, corresponde al primer acercamiento que tiene el estado por incluir el enfoque CTS en el desarrollo de las clases de ciencias naturales. 
Esquema 2 - Relación entre las acciones de pensamiento para el área de ciencias naturales propuestas por el MEN

\begin{tabular}{|c|c|c|c|}
\hline \multicolumn{4}{|c|}{...manejo conocimientos propios de las ciencias naturales } \\
\hline Entorno vivo & Entorno físico & Ciencia, tecnología & sociedad \\
\hline $\begin{array}{l}\text { Esta columna se } \\
\text { refiere a las } \\
\text { competencias } \\
\text { específicas que } \\
\text { permiten } \\
\text { establecer } \\
\text { relaciones entre } \\
\text { diferentes ciencias } \\
\text { naturales para } \\
\text { entender la vida, } \\
\text { los organismos } \\
\text { vivos, sus } \\
\text { interac.iones v }\end{array}$ & $\begin{array}{c}\text { Esta otra se refiere a } \\
\text { las competencias que } \\
\text { permiten la relación } \\
\text { de diferentes ciencias } \\
\text { naturales para } \\
\text { entender el entorno } \\
\text { donde viven los } \\
\text { organismos, las } \\
\text { interacciones que se } \\
\text { establecen y explicar } \\
\text { las transformaciones } \\
\text { de la materia }\end{array}$ & $\begin{array}{c}\text { Y esta se refiere a las } \\
\text { competencias } \\
\text { específicas que permiten } \\
\text { la comprensión de los } \\
\text { aportes de las ciencias } \\
\text { naturales para mejorar la } \\
\text { vida de los individuos y } \\
\text { de las comunidades, así } \\
\text { como el análisis de los } \\
\text { peligros que pueden } \\
\text { originar los avances } \\
\text { científicos }\end{array}$ & $\begin{array}{l}\text { Se incorporó, } \\
\text { a la } \\
\text { enseñanza } \\
\text { de las } \\
\text { ciencias, la } \\
\text { dimensión } \\
\text { ambiental } \\
\text { como } \\
\text { elemento } \\
\text { central } \\
\text { configurándo } \\
\text { se la } \\
\text { Fdı ırarińn }\end{array}$ \\
\hline
\end{tabular}

Tomado de los estándares curriculares formulados por el Ministerio de Educación Nacional en la guía $\mathrm{N}^{\circ}$ 7 (MEN, 2006).

En el esquema 3, se puede observar que el eje disciplinar (conocimientos propios de la ciencia), propone vincular el enfoque CTS a través de una serie de acciones que se deben abordar en los diferentes niveles académicos, para que así se puedan alcanzar los distintos estándares curriculares propuestos para el área. Desafortunadamente en ese documento no se encuentra una definición conceptualizada de lo que se entiende o se debe entender por el enfoque CTS. Al no contar con esta definición, el análisis de las acciones de pensamiento es la base para conceptualizar la concepción de CTS del currículo (BAQUERO, 2014). Además, el MEN, en su propuesta de CTS para los distintos grados escolares, espera que tanto estudiantes como docentes desarrollen concepciones o visiones de estas complejas relaciones a lo largo de su vida escolar (BAQUERO, 2014).

Esquema 3 - Estructura de la enseñanza de las ciencias naturales en Colombia.

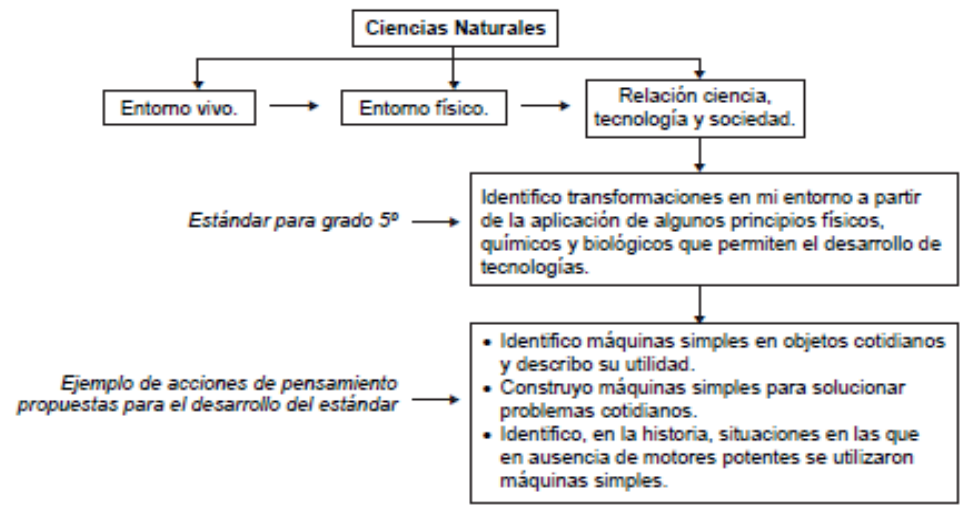

Tomado de (MEN 2006). Adaptación del eje disciplinar de los estándares nacionales de ciencias naturales, para mostrar sus componentes y ejemplos de acciones de pensamiento CTS. 
En el esquema 3 se puede observar el planteamiento que hace el MEN en torno a la organización conceptual de la enseñanza de las ciencias naturales, las cuales divide en tres componentes que son: entorno vivo (Biología), entorno físico (Física) y relación CTS. Es de aclarar que en los estándares curriculares se encuentra discriminado cada uno de los componentes por cada nivel del conocimiento, sin embargo, para interés de esta investigación, solo se hace referencia a la inclusión de los estándares y las acciones de pensamiento que propone el MEN. En torno al enfoque CTS, esas relaciones dan evidencia de lo propuesto por Ziman (1980) frente a las tendencias de la enseñanza de las ciencias desde el enfoque CTSA.

Es evidente que la intención del MEN, con respecto a la inclusión del enfoque CTS en los diferentes niveles escolares, es que los estudiantes, con el apoyo de los profesores, desarrollen a lo largo de la vida escolar competencias y visiones de la relación entre la Ciencia, Tecnología y la Sociedad.

Con respecto a la inclusión del componente ambiental en el enfoque CTS, es necesario resaltar que el Estado colombiano, por medio de la articulación entre el MEM y el Ministerio de Medio Ambiente, emitió, en 1994, el decreto 1743, que referencia el proyecto de educación ambiental a nivel nacional. Este se instituye para todos los niveles de educación formal, de igual forma, fija los criterio para la promoción de la educación ambiental formal e informal (MEN, 1994).

Sin embargo, es hasta el 2004, con la publicación de los estándares curriculares, cuando se logran sistematizar los lineamientos curriculares para la educación en ciencias y, específicamente, desde el componente ambiental. De esa manera, se incluyen dos elementos: el enfoque CTSA y la educación ambiental. En el siguiente apartado, se va a profundizar sobre los fundamentos y elementos conceptuales del enfoque CTSA.

\section{Enseñanza de las ciencias en el sector provincial desde el enfoque CTSA}

La enseñanza de las ciencias en las instituciones educativas de básica media debe atender diferentes elementos como lo son las propuestas realizadas desde las entidades del Estado, las características propias de la institución, las políticas educativas, el contexto de los estudiantes y las particularidades de los maestros, entre otras variables. En ese sentido, la inclusión del enfoque CTSA en el diseño curricular, como se ha resaltado en los apartados anteriores, requiere de un ejercicio de aceptación, adaptación y ejecución por parte de los docentes especialistas en el área de cada una de las instituciones. En ese sentido, este apartado tiene la intención de identificar las características más relevantes frente a la enseñanza y la evaluación, desde el enfoque CTSA, retomando algunos aspectos mencionados por Vásquez e Rodriguéz (2014) en su artículo "Enseñanza, aprendizaje y evaluación en la formación de docentes en educación CTS en el contexto del siglo XXI".

A través de la historia han surgido varios modelos y teorías sobre la enseñanza, las cuales tienen diferentes fundamentos teóricos, contextuales e históricos. Algunos de los modelos más representativos han sido las teorías conductistas, las explícitas e implícitas, las del desarrollo cognitivo, las del procesamiento de la información o las sociopsicológicas son algunas de las más importantes (ANDERSON, 1995). Por otro lado, han surgidos modelos de enseñanza tales como la instrucción directa, el aprendizaje cooperativo, el aprendizaje por descubrimiento, la instrucción personalizada, el aprendizaje de dominio, la instrucción en el laboratorio y la enseñanza reflexiva (VÁZQUEZ; RODRIGUÉZ, 2014). 
Cada una de esas teorías y modelos ha aportado elementos conceptuales en la consolidación del enfoque CTSA como una categoría de investigación, sin embargo, como lo menciona Vázquez e Rodriguéz (2014), son la teoría explícita y la enseñanza reflexiva las que se consolidan como el sustento teórico más importante del enfoque, razón por la cual, a continuación, se abordarán los elementos teóricos más relevantes de la teoría explícita y el modelo reflexivo.

Por un lado, la enseñanza explícita tiene como principal objetivo permitir que los estudiantes construyan sus ideas previas, edificándolas desde los diferentes aportes que facilitan los hechos, conceptos, destrezas y otras formas del conocimiento que se dan en un contexto específico (WINNE, 1995).

Las características más importantes de la enseñanza explícita son: la explicación e identificación por parte de los estudiantes de los objetivos de la enseñanza, la revisión de los conocimientos que son requisitos para que el estudiante pueda reunir y elaborar las estructuras de la información que unen las ideas previas con la nueva información presentada. En ese sentido, los nuevos contenidos se presentan en pasos pequeños o pequeños paquetes de información para evitar la sobrecarga de la memoria a corto plazo, lo anterior implica necesariamente explicaciones y demostraciones de reglas que son comprendidas y adaptadas por los estudiantes (VÁSQUEZ; RODRIGUÉZ, 2014). Es de resaltar que las actividades que desarrollan los estudiantes tienen la finalidad de resolver situaciones problémicas o cuestionamientos que permitan aplicar lo aprendido durante las clases.

Por otro lado, la enseñanza reflexiva se define como aquella teoría que favorece el aprendizaje metacognitivo, a través del desarrollo de un análisis crítico de situaciones particulares, que en el mejor de los casos, deben ser contextualizadas. De igual forma, se favorecen destrezas de razonamiento lógico, juicio sensato y actitudes favorables a la reflexión. Bajo esos parámetros, existen algunos contextos que deben ser tenidos en cuenta, a la hora de estructurar las clases de ciencias, basadas en el enfoque CTSA, los cuales se resaltan a continuación (VÁSQUEZ; RODRIGUÉZ, 2014):

a) Aportar un ambiente de apoyo emocional en el aula que fomente la reevaluación de conclusiones;

b) Facilitar tiempo suficiente a los estudiantes para responder preguntas;

c) Revisar la situación de aprendizaje (lo que se conoce, lo que no se conoce y lo que se ha aprendido);

d) Proveer un ambiente abierto o menos estructurado para incitar a los estudiantes a explorar lo que consideren importante;

e) Construir entornos de aprendizaje social (trabajos y actividades en grupos pequeños) para que los estudiantes afronten otros puntos de vista;

f) Proporcionar oportunidades para involucrar a los estudiantes en la recopilación de información para buscar causas y soluciones;

g) Proporcionar ideas y hojas de actividades para ayudar a los estudiantes a evaluar las pruebas que se reúnen;

h) Proporcionar oportunidades de elegir e implementar la mejor alternativa;

i) Animar a vigilar y reevaluar los resultados y conclusiones a lo largo de todo el aprendizaje;

j) Dar sugerencias de andamiaje gradual mediante recursos y hojas de actividades que promuevan la investigación, la curiosidad, la exploración y los procesos de metacognición (lo que saben, lo que han aprendido, lo que necesitan saber). 
Los contextos mencionados por Vázquez e Rodriguéz (2014) aportan en el desarrollo de las clases de ciencias en cualquier espacio territorial. Para el caso específico de la educación en ciencias desde el enfoque CTSA en el sector provincial colombiano, se puede desarrollar actividades que favorezcan la metacognición y que tengan en cuenta el contexto socioeconómico de los estudiantes, tales como:

a) Identificar las características socioeconómicas y culturales de los estudiantes;

b) Determinar las necesidades y expectativas de los estudiantes;

c) Usar el conocimiento cotidiano de los estudiantes y habitantes de la comunidad como un insumo en el desarrollo de las clases;

d) Diseñar estrategias que abran la escuela, es decir que permita que los estudiantes retroalimenten a la comunidad lo aprendido;

e) Involucrar a los estamentos gubernamentales en los procesos de enseñanza aprendizaje de las ciencias;

f) Identificar y atender de manera responsable los problemas que impactan negativamente a la comunidad;

g) Generar espacios de discusión y reflexión en torno a las situaciones municipales, nacionales y globales que afecten a la comunidad.

\section{Consideraciones finales}

Como se ha podido observar, es evidente que existe una deuda, de parte del Estado, con el sector provincial, bien sea desde los espacios investigativos que se consolidan en las comunidades académicas de las universidades, así como desde las diferentes políticas públicas emitidas por el gobierno colombiano. De esa manera, se hace necesario proponer un debate frente al mejoramiento de la calidad educativa en cada uno de los sectores del territorio nacional desde dos escenarios: el primero corresponde a los programas de formación de docentes, en el que es necesario incluir en reconocimiento del sector provincial como un posible escenario laboral de los futuros maestros y, por el otro lado, se hace indispensable que el estado Colombiano formule políticas educativas que atiendan las particularidades del sector provincial.

Un aspecto que puede contribuir a minimizar la deuda con el sector provincial y rural disperso es la proyección de una educación en esos sectores, que trascienda las políticas educativas centralizadas y basadas en la obtención de resultados académicos. Para lograr eso, se invita al Estado a reestructurar esas políticas, articulando el saber y el aprendizaje con las variables sociales, culturales, políticas y económicas que brinda el contexto rural, pero que dentro del proceso pedagógico se propicie en los estudiantes el sentido de pertenencia por el territorio y la cultura.

Para lograr una educación en ciencias pertinente y de calidad, se hace necesario generar espacios de reflexión entre los maestros de educación media, en el que se aborde la importancia que tiene en la enseñanza de las ciencias la relación entre: el desarrollo integral del sector provincial, las problemáticas del mismo y los procesos pedagógicos, razón por la cual se invita a las comunidades de especialistas y al estado colombiano tener en cuenta esos elementos en sus procesos investigativos.

La función de la educación en ciencias en el sector provincial es fundamental en el desarrollo del país. No se puede concebir un programa eficaz de desarrollo rural sustentable si no se forma y capacita al habitante del campo para que cumpla su papel de protagonista y beneficiario de ese desarrollo, el cual debe incluir a los campesinos como pieza fundamental del proceso. 
Los procesos de enseñanza desde el enfoque CTSA no solo deben desarrollar en los jóvenes campesinos las diferentes competencias generales y específicas, sino que el sistema educativo debe propender por desarrollar habilidades que les permitan a los jóvenes cumplir con su rol como campesinos, agricultores y ciudadanos. De igual manera, la enseñanza de las ciencias desde dicho enfoque debe permitir la articulación de los procesos pedagógicos con el sector productivo de las regiones.

Desde luego, todo lo anterior implica una concepción integrada de la formación, de modo que unos aspectos actúen sobre los otros y se refuercen mutuamente. Además, tiene que llevarse a cabo en función directa con las necesidades, intereses y problemas de los habitantes del sector provincial. Por último, esa formación no puede ser estandarizada ya que, en la realidad del sector provincial, existe una gran variedad de situaciones, de ahí que la educación de los estudiantes provinciales deba situarse en el contexto del desarrollo nacional y en una situación en que opere una transformación en los demás aspectos de su vida, siendo ello indudablemente el elemento primordial de los cambios que deben operarse en las comunidades rurales.

\section{Referencias}

ACEVEDO, J. A. Educación tecnológica desde una perspectiva CTS. Alambique: didáctica de las ciencias experimentales, Barcelona, n. 3, p. 75-84, 1995.

ACEVEDO, J. A. la tecnología en las relaciones CTS: una aproximación al tema. Enseñanza de las Ciencias, Barcelona, v. 14, n. 1, p. 35-44, 1996.

ANDERSON, L. International Encyclopedia of Teaching and Teacher Education. Oxford: Pergamon Press, 1995.

BAQUERO, J. Qué visiones de CTS tienen los docentes de $5^{\circ}$ y $9^{\circ}$ grado de Colombia? Y ¿cuál es su relación con los estándares de ciencias del Ministerio de Educación Nacional? Ciênc. educ., Bauru, v. 20, n. 4, 2014.

BERNSTEIN, B. Pedagogía, control simbólico e identidad. Madrid: Morata, 1996.

CASTAÑEDA, A.; ROSAS, A.; MOLINA, J. La institucionalización del conocimiento en la clase de matemáticas. Un estudio sobre el discurso del aula. Perfiles

Educativos, v. XXXIV, n. 135, p. 26-40, 2012.

FALS BORDA, O. Acción y espacio: Autonomías en la nueva República. Bogotá: Tercer Mundo, 2000.

FURIÓ, C.; VILCHES, A. Las actitudes del alumnado hacia las ciencias y las relaciones ciencia, tecnología y sociedad. In: DEL CARMEN, L. (coord.). La enseñanza y el aprendizaje de las ciencias de la naturaleza en la educación secundaria. Barcelona: Horsori, 1997.

GIL PÉREZ, D. et al. A proposal to enrich teachers' perception of the state of the world: first results. Environmental Education Research. Abingdon, v. 9, n. 1, p. 67-90, 2003. 
GRANATA, M.; BARALE, M.; CHADA, M. La enseñanza y la didáctica: aproximaciones a la construcción de una nueva relación. San Luis: Red Fundamentos en Humanidades, 2005.

HERNÁNDEZ, C. Estados del Arte de la Investigación en Educación y Pedagogía en Colombia. Tomo I. Bogotá: ICFES, 2001.

MAIZTEGUI, A. et al. Papel de la tecnología en la educación científica: una dimensión olvidada. Revista Iberoamericana de Educación, Madrid, n. 28, p. 129-155, 2002.

MANSOUR, N. Impact of the Knowledge and Beliefs of Egyptian Science Teachers in Integrating a STS Based Curriculum: A Sociocultural Perspective. Journal of Science Teacher Education, Dordrecht, v. 21, n. 5, p. 513-534, 2010.

MARTÍN, L. Semilleros de investigación. Una estrategia para la formación en ciencias en el sector rural. Colombia: Universidad Pedagógica Nacional, 2009.

MARTÍN, L. Leer la provincia. Alternativas para nuevas prácticas educativas. In: CONGRESO DE TRANSFORMACIÓN EDUCATIVA, 2., 2015, México. Anais [...]. México: Amapsi, 2015.

MARTINEZ, L; ROJAS, A. Estrategia didáctica con enfoque ciencia, tecnología, sociedad y ambiente, para la enseñanza de la bioquímica. Revista TEA, n. 19, p. 44 62, 2006.

MINISTERIO DE EDUCACIÓN NACIONAL. Decreto 1743 de agosto de 1994. Diario Oficial n. 41.476, del 5 de agosto de 1994. Ministerio de Educación Nacional, 1994.

MINISTERIO DE EDUCACIÓN NACIONAL. Serie lineamientos curriculares Ciencias Naturales y Educación Ambiental. Colombia: Ministerio de Educación Nacional, 1998.

MINISTERIO DE EDUCACIÓN NACIONAL. Estándares básicos de competencia en lenguaje, matemáticas, ciencias y ciudadanas. Bogotá, 2006.

PÉREZ, R. et al. La enseñanza de las ciencias experimentales, el constructivismo del caos. Colombia: Mesa Redonda, 1998.

POPHAM, W. J. Problemas y técnicas de la evaluación educativa. Madrid: Anaya, 1980.

SANMARTÍN, J. et al. Estudios sobre sociedad y tecnología. Barcelona: Anthropos, 1992.

SOLBES, J. Les empremtes de la ciència. Germania: Alzira, 2002.

SOLBES, J.; VILCHES, A. Papel de las relaciones entre ciencia, tecnología, sociedad y ambiente en la formación ciudadana. Enseñanza de las Ciencias, Barcelona, v. 22, n. 3, p. 337-348, 2004. 
TORO, J. ¿Qué visiones de CTS tienen los docentes de $5^{\circ}$ y $9^{\circ}$ grado de Colombia? Y ¿cuál es su relación con los estándares de ciencias del Ministerio de Educación Nacional? Ciência. educ., Bauru, v. 20, n. 4, out./dez. 2014.

TORRE, A.; WALLET, F. Regional Development in Rural Areas: Analytical tools and public policies. París: Springer, 2016.

VÁZQUEZ, A.; RODRÍGUEZ, A. Formación del profesorado en naturaleza de la ciencia mediante investigación-acción. Praxis \& Saber, v. 5, n. 9, p. 165 - 188, 2014.

WINNE, H. Information Processing Theories of Teaching. International Encyclopedia of Teaching and Teacher Education. Oxford: Pergamon Press, 1995. p. 107-112.

ZAMBRANO, C. La relación entre conocimiento común y conocimiento científico en el contexto de la enseñanza, aprendizaje y cambio conceptual de las ciencias. Revista TED, n. 3, 1998.

ZIMAN, J. Teaching and Learning about Science and Society. Cambridge:

Cambridge University Press, 1980.

Recebido em maio de 2020.

Aprovado em novembro de 2020. 